nach Transplantation abgesetzt werden. Das Nebenwirkungsprofil entspricht der Kontrollgruppe, allerdings entwickelte keiner der 17 „toleranten“ Patienten einen Posttransplantationsdiabetes. Eine GvHD („graft-versus-host disease“) war bei keinem der 17 Patienten aufgetreten. Das Verfahren soll nun in Stanford für Patienten ohne erhöhtes immunologisches Risiko als „standard of care“ eingesetzt werden.

Kommentar. Im Vergleich zu den bisherigen Protokollen ist die Vorbehandlung wesentlich weniger toxisch und insofern auch eher in die Praxis überführbar. Allerdings sollte nicht unerwähnt bleiben, dass 5 der 22 Patienten (knapp ein Viertel) einer Immunsuppression bedürfen. Das Verfahren bereits jetzt als „standard of care" einzusetzen, halte ich für verfrüht.

\section{Korrespondenzadresse}

\section{Prof. Dr. M. Zeier}

Sektion Nephrologie, Medizinische Klinik I Im Neuenheimer Feld 162, 69120 Heidelberg, Deutschland

Martin.Zeier@med.uni-heidelberg.de

Interessenkonflikt. M. Zeier gibt an, dass kein Interessenkonflikt besteht.

\section{Literatur}

1. De Fijter JW et al (2015) Better renal function preservation with early conversion to Everolimus $n$ DE NOVO renal transplant recipients: 24 -months results from the ELEVATE study. J Am Soc Nephrol 26:76A

2. Budde $\mathrm{K}$ et al (2015) Month 48 follow-up results of the HERAKLES study: superior renal function after early conversion to an everolimus-based calcineurin free regiman. J Am Soc Nephrol 26:76A

3. Vincenti F et al (2015) Belatacept pts. had superior graft survival versus CsA pts: final results from BENEFIT. J Am Soc Nephrol 26:75A

4. Vincenti $F$ et al (2010) A phase III study of belatacept-based immunosuppression regimens versus cyclosporine in renal transplant recipients (BENEFIT study). Am JTransplant 10:535-546

5. Scandling et al (2015) Tolerance induction versus conventional immunosuppression in HLAmatched kidney transplantation: comparison at two years post transplant. JASN 26:75A

Nephrologe 2016 · 1:51

DOI 10.1007/s11560-015-0017-2

(c) Springer-Verlag Berlin Heidelberg 2015

CrossMark

\title{
G. Wolf
}

Klinik für Innere Medizin III, Universitätsklinikum Jena, Jena, Deutschland

\section{Erratum zu: Diabetes und Nierenerkrankungen}

\section{Erratum zu: \\ Nephrologe 2015 5:382-385}

Im Originalbeitrag ist Tab. 1 leider fehlerhaft.

Unter Stadium IIb muss es unter „Schwere mesangiale Expansion“ bei den Kriterien heißen: „Schwere mesangiale Expansion in > $25 \%$ des Mesangiums“ (statt „Milde mesangiale Expansion“ in > $25 \%$ des Mesangiums).

\section{Korrespondenzadresse}

\section{Prof. Dr. G. Wolf}

Klinik für Innere Medizin III, Universitätsklinikum Jena

Erlanger Allee 101, 07740 Jena, Deutschland gunter.wolf@med.uni-jena.de 